\title{
Frontières
}

\section{De la mélancolie considérée comme remède}

\section{Martin Tailly}

Volume 21, numéro 2, printemps 2009

Détresse psychique et antidépresseurs

URI : https://id.erudit.org/iderudit/039457ar

DOI : https://doi.org/10.7202/039457ar

Aller au sommaire du numéro

Éditeur(s)

Université du Québec à Montréal

ISSN

1180-3479 (imprimé)

1916-0976 (numérique)

Découvrir la revue

Citer cet article

Tailly, M. (2009). De la mélancolie considérée comme remède. Frontières, 21(2), 46-50. https://doi.org/10.7202/039457ar

\section{Résumé de l'article}

Cet essai cherche, à partir de cette affirmation - « La mélancolie est peut-être le remède idéal ou le seul utile pour moi, prendre continuellement des comprimés de mélancolie... »-, à définir le rôle de la mélancolie dans la pensée de Thomas Bernhard. Véritable négatif de l'antidépresseur, en ce qu'il ne cherche ni la normalisation de la pensée ni l'uniformisation du rapport à l'Autre, le comprimé de mélancolie a pour but d'induire un effet similaire à la volonté éperdue de lucidité qui guide tous les narrateurs de l'oeuvre de Bernhard, volonté toujours bipolaire de voir le monde dans toute sa contradiction. Considérée à la fois comme médicament de sur-vie et comme dispositif réflexif, autocritique, la mélancolie bernhardienne indique la façon dont l'imperfection de la pensée en est le seul moteur. 


\section{Résumé}

Cet essai cherche, à partir de cette affirmation - "La mélancolie est peut-être le remède idéal ou le seul utile pour moi, prendre continuellement des comprimés de mélancolie...» -, à définir le rôle de la mélancolie dans la pensée de Thomas Bernhard. Véritable négatif de l'antidépresseur, en ce qu'il ne cherche ni la normalisation de la pensée ni l'uniformisation du rapport à l'Autre, le comprimé de mélancolie a pour but d'induire un effet similaire à la volonté éperdue de lucidité qui guide tous les narrateurs de l'œuvre de Bernhard, volonté toujours bipolaire de voir le monde dans toute sa contradiction. Considérée à la fois comme médicament de sur-vie et comme dispositif réflexif, autocritique, la mélancolie bernhardienne indique la façon dont l'imperfection de la pensée en est le seul moteur.

Mots clés: Thomas Bernhard mélancolie - antidépresseur autoréflexivité.

\section{Abstract}

From this statement - "Melancholy is perhaps the ideal remedy or the only useful for me, continually taking tablets of melancholy..." -, this essay strives to define the role of the melancholy in Thomas Bernhard's thinking. An antithesis to antidepressants, as it neither seeks the normalization of thought nor try to standardize the relation to the Other, the melancholy tablet generates an effect similar to the desperate will of lucidity that guide all of Bernhard's narrators: a bipolar will to see the world in all its contradictions. Considered both as a survival medicine and an autoreflective device, melancholy in Bernhard's work shows how the imperfection of thought is the engine of thought itself.

Keywords: Thomas Bernhard melancholy - antidepressant self-criticism.

$\begin{array}{llllllll}\text { A } & R & T & \text { I } & \text { C } & \text { L } & \text { E } & \text { S }\end{array}$

\title{
DE LA MÉLANCOLIE CONSIDÉRÉE COMME REMÈDE
}

\author{
DÈS MA PLUS TENDRE ENFANCE, MA CONFIANCE EN LA VIE \\ S'ÉTAIT BRISÉE AUX IMPRESSIONS SOUS LESQUELLES \\ AVAIT LUI-MÊME SUCCOMBÉ LE MÉLANCOLIQUE VIEILLARD \\ QUI ME LES AVAIT IMPOSÉES : ENFANT, Ô FOLIE ! \\ JE REÇUS LE COSTUME D'UN MÉLANCOLIQUE VIEILLARD.
}

KIERKEGAARD (1971, P. 54).

Martin Tailly,

étudiant à la maîtrise, Département des littératures de langue française, Université de Montréal.

\section{GERME DE GUÉRISON}

Les antidépresseurs, qui apparaissent comme autant de soleils synthétiques à ingérer quotidiennement afin, sinon d'illuminer la nuit profonde de notre existence, au moins de nous rendre indifférents à l'absence de tout autre soleil, viennent pallier ce qui jusqu'à maintenant n'avait jamais été qu'immatériel. Comme si le mensonge vital avait enfin trouvé sa substance, son alchimie concrète. Et sa transposition du domaine de la métaphysique à celui de la physique, ce passage par la chimie pour donner du corps au mensonge ou pour donner du mensonge au corps, ne l'a en rien éloigné de tout ce qui caractérisait la fiction divine ou métaphysique. D'une forme de pouvoir à l'autre, c'est le retour du même - ou presque : on achète maintenant la paix (et son aveuglement) de la même façon qu'anciennement on payait la dîme, on fait encore taire nos angoisses en payant grassement l'hostie spirituelle, on se met dans le même état de dépendance vis-à-vis de la substance et du médecin que vis-à-vis du prêtre et de la foi, devant lesquels on finissait irrémédiablement à genoux.

L'humanité, cette inhumanité, comme la qualifie Bernhard, n'a cessé de consolider le monde sur un arrière-monde que lorsque celui-ci s'est écroulé entraînant le monde entier dans sa chute et dans sa nuit - «Ne fait-il pas nuit sans cesse et de plus en plus nuit? » se demande l'Insensé (Nietzsche, 1973, p. 209). Et maintenant, c'est au monde intérieur, siège même de la conscience du monde extérieur, qu'elle 
tente d'imposer, et ce, au détriment de la vérité, une vision dénuée d'angoisse et de mélancolie, une vision empreinte d'optimisme, une normalisation, un contrôle de la pensée, qui visent à la fois le bon fonctionnement de la société et le bonheur de l'individu (même s'il a effectivement toutes les raisons d'être malheureux) - une utopie médicale étatique autant socialiste que capitaliste. Les soleils factices de la pharmaceutique, les antidépresseurs, en tant que tentative de désaveu du soleil noir de la mélancolie, du réel dans son imperfection, de l'imperfection comme moteur de la pensée, de la pensée en tant qu'elle doit se confronter au réel, uniformisent le rapport à l'Autre, que celui-ci soit intérieur ou extérieur au sujet, s'attaquent à la pensée dans tout le jeu de sa négativité et, par conséquent, entraînent le Propre, qui n'est jamais lui-même que dans le rapport qu'il entretient avec ce qu'il n'est pas, vers l'impropre.

C'est comme ça que cela doit être, c'est comme ça, disent les professeurs [dont le rôle est aussi, de nos jours, incarné par la substance et les médecins], et ils ne tolèrent pas la moindre contradiction, parce que cet État catholique ne tolère pas la moindre contradiction, et ils ne laissent rien à leurs élèves, absolument rien en propre (Bernhard, 1991, p. 47).

En tendant à neutraliser toute contradiction, et en s'en prenant - sans qu'aucun spécialiste ne se soucie de son étiologie véritable - au désespoir comme à quelque chose de pathologique en soi, dans ce qui apparaît comme une véritable chasse aux sorcières, les antidépresseurs, qui étouffent le sujet pensant avec la camisole de force de leur positivité, éloignent le sujet de tout penser lucide et vivant, car, si, comme l'appelle Adorno, le tout négatif, c'est-à-dire la vie dans toute l'insolubilité de ses contradictions, s'assimile davantage à l'aspect bipolaire de la mélancolie - à l'alternance et à la complémentarité de ses phases de manie et de dépression - qu'à la cellule capitonnée et homogène de la conscience intoxiquée, la pensée véritable, à savoir la pensée vivante et lucide doit, elle, revêtir une forme pathologique, "déraisonnable» aux yeux de la «raison dominante», et une

[...] fois qu'elle sait que l'Universel dominant et les proportions qui sont les siennes, sont malades - à proprement parler : atteints de paranoïa et de «projection pathologique»- alors ce qui se présente précisément comme malade, aberrant, paranoïde et même complètement «fou», au regard des critères de cet ordre dominant, c'est pour elle le seul germe de guérison (Adorno, 2003a, p. 99).

UNE TELLE PENSÉE, DANS SA VOLONTÉ ÉPERDUE DE LUCIDITÉ, NE FAIT

PAS QUE DÉVOILER L'ABÎME AUQUEL LES ANTIDÉPRESSEURS ARRIVENT

À NOUS RENDRE INDIFFÉRENTS, MAIS EN SOULIGNE L'IMPORTANCE:

L'IMPORTANCE DE L'IMPERFECTION COMME SEULE RAISON QUI

NOUS POUSSE À CONTINUER - L'IMPERFECTION DE LA PENSÉE

ET DE LA VIE COMME MOTEUR MÊME DE LA PENSÉE ET DE LA VIE.

Ce type de pensée (dialectique) en est, en soi, un de résistance, où l'idée même de guérison, de survie n'a de sens que par rapport à la menace qui pèse sur elle, qui en est inséparable, et ce germe de guérison, qui se doit d'être pathologique pour ne pas l'être, a bien quelque chose de la mélancolie, du soleil noir de la mélancolie, de cela même que veut enrayer l'antidépresseur. Cette pensée de la guérison, d'une survie viable (du sujet et de sa pensée) rendue possible par la mélancolie, d'une survie dite viable s'opposant à la vie aseptisée par la pharmaceutique, vie qui, tout en protégeant, par de nombreux aspects, le sujet de l'angoisse et du désespoir liés à son irrémédiable inadéquation avec le réel et lui-même, à la virtualité de sa mort, de sa mort dans tout ce qu'elle a d'inévitable, n'arrive pas à masquer son air de trépas. Une telle pensée, dans sa volonté éperdue de lucidité, ne fait pas que dévoiler l'abîme auquel les antidépresseurs arrivent à nous rendre indifférents, mais en souligne l'importance: l'importance de l'imperfection comme seule raison qui nous pousse à continuer - l'imperfection de la pensée et de la vie comme moteur même de la pensée et de la vie. Penser, survivre, se mouvoir dans un tel abîme, voilà l'entreprise d'un auteur tel que Bernhard, qui, pince-sans-rire, affirme dans Trois jours que «la mélancolie est peut-être le remède idéal ou le seul utile pour [lui], prendre continuellement de la mélancolie en comprimés...» (2007, p. 33). Il ne s'agit plus ici de fuir l'abîme, la contradiction, mais de s'y maintenir coûte que coûte.

\section{RÉSISTANCES, CONTRAINTE}

Se faire une habitude de la mélancolie, s'en faire une habitude de vie ou, plutôt, de survie et de pensée: prendre continuellement des comprimés de mélancolie, cela équivaut à vouloir, et Bernhard, ironique, est conscient de ce qu'une telle idée prise au sens littéral aurait d'insensé, constamment induire en soi un état d'esprit qui soit propre au penser lucide et non pas à la paix intérieure, non pas à cette paix intérieure dans tout ce qu'elle nécessite d'aveuglement, et, ce faisant, Bernhard insiste sur la disposition réflexive du mélancolique, qui ne s'adonne jamais à la réflexion qu'en vertu du fait qu'il est mélancolique, à savoir parce qu'il ressent le(s) manque(s), l'abîme, l'absurdité de l'existence - «L'homme pensant est par nature un homme malheureux » peut-on lire dans Maîtres anciens (1991, p. 89). De façon similaire, Kierkegaard, dans son Journal, insiste sur le rôle décisif qu'a joué la mélancolie dans le développement de son esprit, et, cette mélancolie, il l'assimile également, quoiqu'il ne le fasse pas de façon aussi patente que Bernhard, à un remède, remède qu'il oppose au mensonge, à l'aveuglement :

Je me suis jeté dans la vie avec une voie d'eau dans la cale depuis le début - et à cet effort même pour me maintenir à flot à coups de pompe je dois d'avoir développé une existence spirituelle hors de pair. Ça m'a réussi. J'ai interprété cette souffrance comme une écharde dans ma chair [cette écharde dans sa chair à propos de laquelle Kierkegaard écrit, ailleurs dans le Journal, qu'elle n'est autre qu' «une terrible mélancolie» (Kierkegaard, 1957, p. 94)], et j'ai reconnu ce hors de pair au cuisant de l'écharde et le cuisant de l'écharde à ce hors de pair. Tel me suis-je compris moi-même. Autrement j'aurais dû tâcher d'aveugler un peu l'avarie. [...] La contrainte est dans ces cas-là l'unique chose qui aide, car l'infini est une puissance trop grande pour pouvoir servir seule de remède en pareil cas (Kierkegaard, 1954, p. 132).

Mais, alors que chez Kierkegaard elle travaille de pair avec l'infini et le tout, qu'elle en est même la marque, qu'elle est non pas comprimés servant à pallier sa propre intermittence, mais écharde dans la chair, c'est-à-dire à la fois remède et présence continue d'une douleur incarnée, d'une douleur à la fois physique et morale rappelant à l'homme ses limites ${ }^{1}$, et qu'elle tire son origine de l'humilité, de ce sentiment d'insuffisance imposé à l'homme par le divin (Deuxième Épître de saint Paul aux Corinthiens, 12, 7-9), la mélancolie est, chez Bernhard, "le remède idéal, le seul utile» : non seulement elle s'oppose 
«au tout et au parfait», lequel «menace sans arrêt de nous détruire» (1991, p. 89), et à ce qui se donne à voir comme tout et parfait - et pas seulement au sens divin de ces termes, qui, du haut de leur toutepuissance, semblent d'ailleurs craindre la faiblesse et l'imperfection, et cherchent à tout prix, comme on le ferait avec un témoin gênant, à bâillonner l'angoisse et le désespoir du mélancolique -, mais elle en désigne le mensonge. C'est ainsi le passage par l'imperfection, par la pathologie que représente la mélancolie, qui permet au sujet de se guérir de sa manie du «tout positif » et de la perfection, et d'en débusquer le mensonge en toute occasion: la mélancolie, un remède idéal contre tout idéal.

En elle, en la mélancolie, comme dans la cale kierkegaardienne, toujours le risque de sombrer, d'être submergé, ou de s'y abandonner complètement - m'en est témoin le nombre de suicidés portés par la fiction bernhardienne -, mais penser et survivre, entendus comme un seul et même mouvement de résistance, n'est possible qu'au prix d'une exposition à ce risque, à cette contrainte, à cette résistance. La contrainte est au penser kierkegaardien ce que sont les résistances à la pensée de Bernhard: un mal nécessaire. De fait, Bernhard affirme dans Trois jours que "le cerveau a besoin de résistances» et que c'est ainsi à partir de leur rapport aux résistances que se différencient l'homme pensant et l'imbécile, pour lequel la réflexion est impossible, attendu que, pour lui, qui, par sa cécité, c'est-à-dire par sa condition d'imbécile, n'en peut avoir conscience, «il n'y a pas de difficultés » (2007, p. 28), pas de résistances. Penser constitue, tout comme le fait d'écrire, un acte de résistance, un acte de résistance aux résistances en tant qu'elles sont perçues, vécues ou, simplement, entrevues. Et ces résistances, il serait tout à fait faux de croire qu'elles ne proviennent que de l'extérieur ou, même, de l'ordre dominant - ordre dominant, qui, que ce soit sous une forme édulcorée ou non, finit par prendre part à la composition du surmoi et, par conséquent, à être intériorisé -, car penser, c'est s'opposer à soi-même ${ }^{2}$, se faire violence, et faire violence à sa volonté sinon de plaisir, du moins à sa volonté d'éviter le déplaisir ${ }^{3}$, volonté qui, de façon analogue à la pulsion de destruction, semble tendre à la suppression des tensions internes, au bonheur non pas «anorganique», mais végétatif: «On ne veut aucun livre, on ne veut aucune pensée non plus, on ne veut ni langue ni mots, pas de phrases, on ne veut aucune histoire - on ne veut [notre soulignement] absolument rien » (2007, p. 28).

\section{SURVIVANCE ET MÉLANCOLIE}

Le penser mélancolique que d'aucuns considèrent, et c'est là l'idée même de Freud, comme un penser ayant plutôt trait à la pulsion de mort qu'à la pulsion de vie, alors que sa vérité serait, de préférence, à chercher dans l'alliage de ces deux pulsions, entre en conflit avec cette volonté d'indolence, avec ce principe de passivité, qui s'oppose en tout au mouvement de résistance incessant du penser mélancolique, par l'entremise duquel le Propre, afin de devenir autre, de sur-vivre, ce qui se fait non sans douleur - « [penser] est exténuant, destructeur » dit Reger dans Maîtres anciens (Bernhard, 1991, p. 154) -, s'expose, court le risque de sombrer complètement dans l'Autre, dans la folie. Le Meurs et deviens de Goethe (1984, p. 43-44) correspond à l'impulsion du devenir autre, qui, à certains égards, et, dans un geste dérisoire, pour embrouiller et complexifier tout à la fois la théorie freudienne des pulsions et son manichéisme, mériterait bien le nom de "pulsion de sur-vie», en ce que la pulsion de vie dont il est question ici procède directement de la pulsion de destruction, qui à son tour... Et ce mouvement incessant qui, malgré les apparences, n'a rien d'une «sursomption» (Aufhebung), et ce, parce que cette dialectique de l'identité, comme le remarque Adorno, ne ramène pas le sujet à ce qu'il n'a jamais été, c'est-àdire à l'unité suprême, libérée de toute contradiction, ce mouvement incessant, disais-je, en tant qu'il est autoréflexif: qu'il s'oppose à soi-même, et c'est bien là le mot d'ordre de la pensée mélancolique bernhardienne - "pourquoi en suis-je venu à écrire, pourquoi est-ce que j'écris des livres? Par opposition à moi-même soudain [souligné par Bernhard], et à cet état [à la haine qu'enfant déjà, il éprouvait pour les livres] - parce que les résistances, je l'ai déjà dit, sont tout pour moi » (2007, p. 31) -, ce mouvement incessant, par son opposition au soi et à son penser, est autocritique, et de telle façon que le sujet de ce penser «se [critique] sans cesse dans une perte des objets critiquable» (Mavrikakis, 2005, p. 17), s'abîme dans cette pulsion de sur-vie.

Au sujet de la propension du mélancolique à l'autocritique, Freud écrit :

Dans certaines de ses autres plaintes contre lui-même, [le mélancolique] nous semble également avoir raison, et ne faire que saisir la vérité avec plus d'acuité que d'autres personnes qui ne sont pas mélancoliques. Lorsque dans son autocritique exacerbée, il se décrit: comme mesquin, égoïste, insincère, incapable d'indépendance, comme un homme dont tous les efforts ne tendaient qu'à cacher les faiblesses de sa nature, il pourrait bien, selon nous, s'être passablement approché de la connaissance de soi, et la seule question que nous nous posons, c'est de savoir pourquoi l'on doit commencer par tomber malade pour avoir accès à une telle vérité (1968, p. 153).

Énigmatique pour Freud, cette pensée d'une maladie comme possible accès à la vérité (comme seule possibilité de combattre l'ordre dominant?) alors que luimême, Freud, dont la profession a consisté, en tant que médecin, à guérir des patients, c'est-à-dire à les normaliser de façon à ce qu'ils puissent réintégrer le bon fonctionnement de la société viennoise ${ }^{4}$, n'a pas manqué de percevoir dans la plupart des maladies et des névroses de ses patients les symptômes d'un conflit ambivalentiel souvent causé par l'hypocrisie de cette même société, comme il n'a pas manqué non plus de rechercher à partir de ces symptômes une vérité demeurée jusque-là irreprésentable aux yeux du sujet. Ainsi, pris entre sa société et cela même qu'il découvrit à son propos, entre sa société et la signification des maux dont les sujets se voyaient atteints, Freud lui-même ne peut échapper à l'ambivalence: "Dans la mesure où il reste encore un ennemi de l'hypocrisie, après tant d'autres, sa position est ambiguë5 , entre la volonté d'une émancipation sans fard de ce qui est réprimé et l'apologie d'une répression sans fard» (Adorno, 2003a, p. 80). La suspicion de Freud envers la mélancolie semble provenir du fait que cette vérité de la mélancolie ne subit aucune déformation, n'est soumise à aucune censure, qu'elle parvient de façon intacte - sans être refoulée dans l'inconscient - à la conscience, et qu'en quelque sorte, ce qui permettrait de caractériser le mélancolique, ce serait non ses agissements en tant qu'ils lui échappent, mais cette conscience qu'il a de soi et d'être mélancolique, cette même "autoconscience» mélancolique à partir de laquelle Bernhard et Kierkegaard affirment en arriver à penser lucidement, mais Freud, qui ne remet pas en cause l'aspect pathologique de la mélancolie, et pour qui la névrose et la véritable conscience de soi sont étrangères l'une à l'autre, cherche l'origine de l'autocritique en dehors de la sphère du moi, en dehors de la conscience, de ce qui est donné, et l'impute ainsi à un objet d'amour perdu - mais inconscient -, dont l'«ombre, tombé sur le moi » (Freud, 1968, p. 158), permettrait à celui-ci de se critiquer (ou au surmoi de considérer le moi) comme s'il était cet objet, faisant ainsi de l'autocritique du mélancolique une illusion, une critique dont certains points parviendraient, de façon complètement fortuite, au général. 


\section{L'ARTISTE CRITIQUE}

Inextricablement liée au deuil - presque pas une œuvre de Bernhard sans morts ou suicidés, presque pas une œuvre qui n'en soit le tombeau -, inextricablement, disaisje, et ce, au sens même où, si Freud écrit que «dans le deuil le monde est devenu pauvre et vide, [et que] dans la mélancolie c'est [apparemment, faut-il ajouter] le moi lui-même » (Freud, 1968, p. 152), c'est à la fois le monde et le moi qui, dans l'œuvre de Bernhard, sont absurdes, pauvres et vides. La mélancolie bernhardienne n'a pas cette univocité de mouvement ni ce caractère illusoire de la mélancolie freudienne: l'autocritique s'y fait réellement autocritique - «ne m'intéressent que mes propres processus» (Bernhard, 2007, p. 31; souligné par l'auteur) - et fait de la contradiction sa nécessité - «seuls les hommes m'ont toujours intéressé, parce que, par nature, ils me repoussent ${ }^{6}$ » (Bernhard, 1991, p. 85) -, elle inaugure un mouvement conscient de lui-même, un mouvement d'aller-retour incessant qui, parce que le sujet ne peut se saisir que dans sa relation à l'Autre, et que l'Autre, lui-même, n'est saisi qu'à partir de cette conscience de soi propre au sujet, ne manque pas de renverser, de façon à tenter, même si le sujet se sait irrémédiablement « réduit aux suppositions » (Bernhard, 1987, p. 35), d'en vérifier la justesse, l'autocritique en critique de l'Autre et la critique de l'Autre en autocritique, toujours dans cette même volonté éperdue de lucidité en vertu de laquelle le sujet bernhardien se fait névrosé pour ne pas l'être, se maintient coûte que coûte dans la mélancolie et «[...] a besoin d'une force surhumaine pour ne pas [et] guérir» (Horkheimer et Adorno, 1983, p. 260) et sombrer:

Ils voyaient bien : je suis l'observateur, l'ignoble individu qui s'est confortablement installé dans le fauteuil à oreilles et s'adonne là, profitant de la pénombre de l'antichambre à son jeu dégoûtant qui consiste plus ou moins à disséquer [souligné par Bernhard], comme on dit, les invités auersbergeriens. Ils m'en avaient toujours voulu de les avoir disséqués en toute occasion, effectivement sans le moindre scrupule, mais toujours avec une circonstance atténuante; je me disséquais moi-même encore bien davantage, ne m'épargnais jamais, me désassemblais moi-même en tous mes éléments constitutifs [...] Et après cela, ce qui restait de moi était encore bien moins de chose que ce qui restait d'eux (Bernhard, 1997, p. 63).

Observateur de soi et des autres, le protagoniste bernhardien est, et il se sait tel, « un artiste critique» (Bernhard, 1991, p. 88), ou, plutôt, un artiste «mélancritique», qui, comme tout artiste, pratique son «art de survie» (Bernhard, 1991, p. 247). Il a ceci de commun avec Marx que son art, sa critique, "n'est pas qu'un [inoffensif] scalpel anatomique, mais une arme» (Marx, 1998, p. 13). Mais dans son cas, l'arme est à double tranchant: le met en pièces, le dissèque encore bien davantage que son objet, et comme il ne dédaigne pas à se faire lui-même objet de sa critique... La pensée mélancritique bernhardienne, en tant qu'elle est art de survie, procède du même mouvement que la pulsion de sur-vie: si elle est destructrice, perte de l'immédiateté, introspection faisant se conjoindre les temporalités, exposition au désespoir, il n'en demeure pas moins qu'elle est aussi devenir autre, sur-vie, mouvement incessant de résistance - de façon similaire à ce que décrit Adorno dans la Dialectique négative, où l'autoréflexivité apparaît comme solution à l'autodestruction de la Raison (Aufklärung), c'est aussi l'autoréflexivité du penser mélancritique bernhardien qui semble préserver le sujet pensant de son anéantissement. Et qu'à la lumière sombre de ce penser, Reger puisse à la fois affirmer, dans Maîtres anciens, que "penser est [...] destructeur» (Bernhard, 1991, p. 154) et modifier de façon suivante le cogito cartésien : "je pense, donc je vis» (1991, p. 117), cela n'a plus rien d'étonnant. Survivre abîme et tue, et plus d'une fois. Survivre abîme et tue, incessamment... jusqu'à ce que survivre ne soit plus possible.

Permettez-moi d'insister : la mélancolie est «médicament de survie» (Bernhard, 1991, p. 234) dans la mesure exacte où l'excitation critique, la Passion critique est son effet. Soulignant l'imperfection de l'homme, les comprimés de mélancolie misent sur son anthropocentrisme. $\mathrm{Ou}, \mathrm{du}$ moins, sur ce qui lui reste d'idéal humaniste. Sur le fait que, pour reprendre les mots de Feuerbach - eux-mêmes repris par Marx -, «l'homme est pour l'homme l'être suprême» (Marx, 1998, p. 25), et que, si, dans son désespoir, il se révèle fragmentaire, et qu'il est, de surcroît, conscient de l'être, c'est-à-dire conscient de son « défaut rédhibitoire », comme le désigne Bernhard (1991, p. 36), de ce défaut par l'entremise duquel ses limites se marquent, l'être suprême ne pourra dès lors plus se considérer comme un être suprême. C'est ainsi que se produit la fracture de son idéal, que, spectateur de sa propre impuissance - et, en ce sens, la Seconde Guerre mondiale s'est faite "comprimés de mélancolie» pour plus d'une génération -, l'homme réalise ce qu'il savait déjà mais avait toujours voulu oublier : il réalise maintenant comme si, s'étant préalablement affranchi du joug de sa création divine, il avait sou- dain cru en détenir le pouvoir - que, pas plus que Dieu, il n'est un tout et parfait, que, s'il est lui-même son véritable soleil - s'il se veut tel -, il s'assimile davantage à un soleil noir qu'au soleil fantasmé et plein, et il prend pleinement conscience du danger que représente pour lui la plénitude, que celle-ci soit incarnée par Dieu, par l'homme ou par une institution: "Pas de plénitude sans manie de la puissance», écrit Adorno dans la Dialectique négative (2003b, p. 458). Le mélancolique bernhardien est cet homme qui, parce qu'il sait que «la perfection menace sans arrêt de nous détruire»(Bernhard, 1991, p. 36), n'a pas seulement en horreur «le tout et la perfection », mais cherche aussi - parce que «c'est seulement lorsque nous nous sommes rendu compte, à chaque fois, que le tout et la perfection n'existent pas, que nous avons la possibilité de continuer à vivre» (1991, p. 37) - à en désigner «le défaut rédhibitoire». Ainsi seulement en arrive-t-il à faire de la mélancolie, de la critique son œuvre de résistance, son «art de survie».

Affranchissement de toute plénitude, remède idéal contre tout idéal, remède contre lui-même, remède autocritique - en ce qu'il exprime la nécessité de l'idéal tout en la critiquant -, "médicament de survie», donc falsification, le penser mélancritique bernhardien est en soi antitotalitaire, tout simplement le négatif de l'antidépresseur.

\section{Bibliographie}

ADORNO, T.W. (2003A). Minima moralia, Paris, Payot, coll. «Petite Bibliothèque Payot », 368 p.

ADORNO, T.W. (2003B). Dialectique négative, Paris, Payot, coll. "Petite bibliothèque Payot », 544 p.

BERNHARD, T. (1987). "Amras», dans Amras et autres récits, Paris, Gallimard, coll. «Du monde entier», p. 9-76.

BERNHARD, T. (1991). Maîtres anciens, Paris, Gallimard, coll. «Folio », 253 p.

BERNHARD, T. (1997). Des arbres à abattre, Paris, Gallimard, coll. «Folio », 232 p.

BERNHARD, T. (2007). "Trois jours", dans Récits 1971-1982, Paris, Gallimard, coll. «Quarto», p. 23-35.

CHESTOV, L. (1998). Kierkegaard et la philosophie existentielle (Vox clamatis in deserto), Paris, Vrin, coll. "Bibliothèque d'histoire de la philosophie», 384 p.

FREUD, S. (1968). «Deuil et mélancolie», dans Métapsychologie, Paris, Gallimard, coll. «Idées », p. 147-174.

GOETHE, J.W. (1984). «Bienheureux désir", dans Le divan, Paris, Gallimard, coll. «Poésie», 245 p.

HORKHEIMER, M. ET T.W. ADORNO (1983). La dialectique de la raison, Paris, Gallimard, coll. «Tel», 281 p. 
KIERKEGAARD， S. (1954). Journal (Extraits), Tome II, Paris, Gallimard, coll. «Les essais», 405 p.

KIERKEGAARD, S. (1957). Journal (Extraits), Tome IV, Paris, Gallimard, coll. «Les essais » 475 p.

KIERKEGAARD, S. (1971). "Point de vue explicatif de mon œuvre d'écrivain ", dans Euvres complètes, Volume 16, Paris, L'Orante, $371 \mathrm{p}$.

MAVRIKAKIS, C. (2005). Condamner à mort, Les meurtres et la loi à l'écran, Montréal, Presses de l'Université de Montréal, coll. «Champ libre», 161 p.

MARX, M. (1998). Contribution à la critique de la philosophie du droit de Hegel, Paris, Éditions Allia, 48 p.

NIETZSCHE, F. (1973). Le gai savoir, Paris, Éditions 10/18, 439 p.

\section{Notes}

1. «J'ai accepté cette triste anomalie et ces souffrances (elles auraient poussé au suicide la plupart des hommes capables de concevoir toute la torture de cette misère), comme une écharde mise dans ma chair, comme ma limite, comme ma croix, comme le prix immense auquel Dieu m'a vendu une force spirituelle qui n'a guère d'égale parmi mes contemporains. » (Kierkegaard, Journal, cité par Léon Chestov, 1998, p. 54.)
2. Voici deux exemples de cette opposition à soi nécessaire à la pensée: "[Méditant] sur l'histoire, sur la nôtre et sur celle des autres, la générale, la grande histoire qui nous rendait fous, sur ces millions de tempêtes de neige faites d'événements et d'évolutions nous avions toujours aimé ce qui nous était pénible et détesté ce qui nous était aisé» (Bernhard, 1987, p. 11) ; "Je suis écœuré par tout ce qu'ils [les maîtres anciens] ont peint et qui est accroché ici, et pourtant depuis des décennies, $[. .$.$] je ne peux pas m'empêcher de$ l'étudier. » (Bernhard, 1991, p. 56.)

3. Il ne faut pas sous-estimer le plaisir qu'éprouve le sujet dans cette opposition à lui-même.

4. Et c'est bien cette même tendance répressive de la société, sa tendance vers la standardisation, la normalisation de l'homme qu'Adorno, dans les fragments trente-six, trente-sept et trente-huit de Minima moralia, reproche aussi à la psychanalyse et à Freud: "Si quelque chose comme une psychanalyse de la culture typique de notre temps était possible - si la prédominance complète de l'économie ne rendait pas dérisoire toute tentative pour expliquer l'état des choses à partir de la psychologie de ceux qui en sont les victimes et si les psychanalystes n'avaient pas euxmêmes fait serment d'allégeance à cet état de choses - une telle recherche aurait à mettre en évidence que c'est dans la normale que réside la maladie de l'époque» (2003a, p. 76);
«Comme spécialiste en matière de psychologie, il [Freud] reprend à son compte l'opposition entre "social" et "égoïste" de façon statique [c'est-à-dire en en privilégiant le pôle social] et sans la remettre en question. Il ne reconnaît pas en elle l'œuvre de la société répressive, pas plus que les traces des mécanismes pernicieux qu'il a lui-même identifiés » (2003a, p. 80$)$; « [...] pour y prendre part [au bonheur sur ordonnance, au bonheur prescrit par les psychanalystes], le névrosé ainsi rendu "heureux" doit abandonner jusqu'à la dernière miette de raison qu'ont pu encore lui laisser le refoulement et la régression, et pour faire plaisir à son psychanalyste, il lui faut s'extasier sans discernement en allant voir des films pornos et en mangeant de la mauvaise cuisine aux pris exorbitants des "restaurants français", en buvant sec et en faisant l'amour dans les limites hygiéniques de ce qui s'appelle maintenant "le sexe" » (2003a, p. 82-83).

5. Cette position ambiguë, c'est toujours au détriment du particulier qu'elle s'affirme. La volonté de libération, du fait que, comme le souligne Adorno, elle ne remet pas en cause le rapport entre la société et l'individu, coïncide avec une répression plus grande encore, et ce, malgré toute la bonne volonté de Freud.

6. Cette affirmation ne fait pas que contredire la précédente, elle-même est opposition.

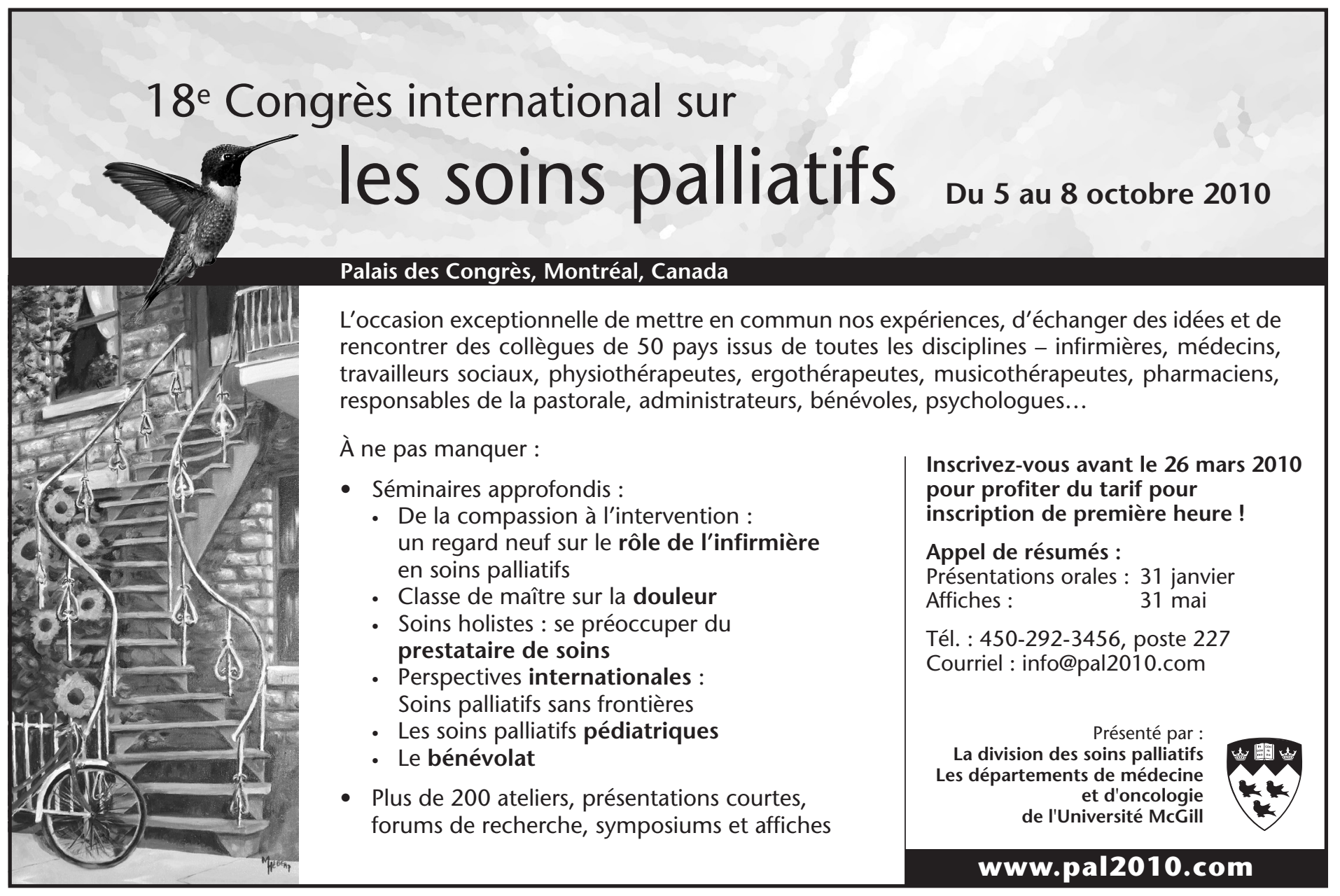

